\title{
Utilização de suplementos alimentares por praticantes de atividade física
}

\author{
Use of dietary supplements by physical activity practitioners \\ Uso de suplementos dietéticos por practicantes de actividad física
}

Recebido: 25/01/2021 | Revisado: 26/01/2021 | Aceito: 31/01/2021 | Publicado: 07/02/2021

Priscylla Tavares Almeida

ORCID: https://orcid.org/0000-0002-9983-988X

Centro Universitário de Juazeiro do Norte, Brasil

E-mail: priscylla_tavares12@hotmail.com

Ygor Teixeira

ORCID: https://orcid.org/0000-0001-6886-9392 Instituto Federal de Educação, Ciência e Tecnologia do Ceará, Brasil

E-mail: positivey@hotmail.com

Naila Thailane Queiroz dos Santos ORCID: https://orcid.org/0000-0003-0312-0318

Centro Universitário Estácio da Bahia, Brasil E-mail: nutrinailaqueiroz@gmail.com

Michele Amaro Tavares

ORCID: https://orcid.org/0000-0002-4360-1880 Centro Universitário de Juazeiro do Norte, Brasil E-mail: michelenutri7@gmail.com

Leilane de Oliveira Azevedo Sobreira ORCID: https://orcid.org/0000-0002-3451-8153

Centro Universitário Estácio de Sá, Brasil E-mail: leilanesobreira@gmail.com

Rainara de Souza Oliveira ORCID: https://orcid.org/0000-0003-4278-3472

Universidade Federal do Amazonas, Brasil E-mail: rainaraoliveeira06@gmail.com

Yasmin Trindade Evangelista de Araújo ORCID: https://orcid.org/0000-0001-9333-0356 Universidade Potiguar, Brasil E-mail: yasmintrindade_@hotmail.com

Ana Karine Gomes de Figueiredo ORCID: https://orcid.org/0000-0002-4305-0847 Universidade Federal da Paraíba, Brasil E-mail: ana.kgfigueiredo@gmail.com

Cícero Jordan Rodrigues Sobreira ORCID: https://orcid.org/0000-0003-0872-5199 Centro Universitário de Juazeiro do Norte, Brasil

E-mail: jordan.nutricionista@hotmail.com

Carla Maria Bezerra de Menezes ORCID: https://orcid.org/0000-0002-1847-9975 Universidade Federal de Pernambuco, Brasil

E-mail: carlamenezes.nutri@ hotmail.com

Laís Amorim Queiroga Carneiro da Cunha ORCID: https://orcid.org/0000-0001-5960-2813

Universidade Federal de Pernambuco, Brasil

E-mail: laisqueiroga@hotmail.com

Samara Chaves de Lima

ORCID: https://orcid.org/0000-0001-6320-0622

Universidade Potiguar, Brasil

E-mail: samara92_chaves@hotmail.com

Brunna da Cruz Araújo

ORCID: https://orcid.org/0000-0003-2653-4011 Centro Universitário Estácio de Sá, Brasil

E-mail: brunna_cruz@outlook.com

Laurineide Rocha Lima

ORCID: https://orcid.org/0000-0003-0908-6210 Universidade Federal do Piauí, Brasil

E-mail: laurineidelima@gmail.com

Priscille Fidelis Pacheco Hartcopff

ORCID: https://orcid.org/0000-0001-9368-6520

Centro Universitário Uniamérica, Brasil

E-mail: priscillepacheco@bol.com.br 
Esaú Nicodemos da Cruz Santana ORCID: https://orcid.org/0000-0002-2417-8706 Universidade de Pernambuco, Brasil E-mail: esausantana@gmail.com

Luciana Nunes de Sousa

ORCID: https://orcid.org/0000-0001-8968-5066 Universidade Regional do Cariri, Brasil E-mail: lucianag888@gmail.com

Paulina Nunes da Silva

ORCID: https://orcid.org/0000-0002-0270-2992 Centro Universitário Uniero, Brasil E-mail: paulina.nutricao@gmail.com

\begin{abstract}
Resumo
Introdução: Um dos aspectos que tem definido a sociedade de consumo é a crescente importância atribuída à imagem corporal. Com isso, consolidou-se nas diversas faixas etárias a busca pela prática de atividades físicas em academias nas suas diversas modalidades. Sabe-se que a ingestão adequada de nutrientes está relacionada com a melhora do rendimento do organismo frente ao desempenho esportivo. Em busca de potencializar resultados nos exercícios os praticantes são conduzidos à procura de recursos com o intuito de alcançar o objetivo a curto prazo. Dentre esses recursos se destacam os suplementos alimentares, estes podem ser adquiridos facilmente, sendo muitas vezes utilizados de forma indevida com o propósito ergogênico e puramente estético. Objetivo: avaliar, através de uma revisão, a prevalência do uso da suplementação alimentar por usuários de academias. Método: Trata-se de uma revisão sistemática acerca do uso de suplementos alimentares em frequentadores de academia realizada na Medical Literature Analysis and Retrieval Sistem On-line (MEDLINE/PUBMED e Google acadêmico, utilizando os termos: suplementos alimentares, atividade física, imagem corporal. A seleção respeitou critérios de inclusão/exclusão previamente elencados. Resultados: Foram selecionados 28 artigos, dentre eles 12 cumpriram aos requisitos. Os estudos mostram acerca da prevalência do uso suplementos por usuários de academias são maiores em homens do que em mulheres. O principal motivo observado pela utilização de suplementos alimentares foi a busca pela hipertrofia muscular, sendo os produtos proteicos os mais consumidos dentre os suplementos, no entanto, observaram-se que a prescrição de tais suplementos não é realizada por profissionais habilitados, sendo os instrutores de academia, seguido de amigos, vendedores de lojas, auto prescrição e por último nutricionistas. Observou-se que os maiores consumidores de suplementos alimentares são praticantes de musculação, visto que é uma modalidade que mescla o desenvolvimento muscular como para melhores resultados estéticos. Conclusão: Portanto, a fim de diminuir o uso indevido, se faz preciso a presença de profissional especializado para atuar em academias ou em locais em que se pratique exercícios físicos, fornecendo orientações nutricionais a fim de melhorar o grau de informação e garantir segurança na utilização desses produtos.
\end{abstract}

Palavras-chave: Exercício físico; Suplementos alimentares; Nutrição; Musculação.

\begin{abstract}
Introduction: One of the aspects that has defined the consumer society is the growing importance attributed to body image. With this, the search for the practice of physical activities in gyms in its different modalities was consolidated in the various age groups. It is known that the adequate intake of nutrients is related to the improvement of the body's performance in relation to sports performance. In order to maximize results in the exercises, practitioners are led to seek resources in order to achieve the short-term goal. Among these resources, dietary supplements stand out, they can be easily acquired, and are often used improperly for ergogenic and purely aesthetic purposes. Objective: to evaluate, through a review, the prevalence of the use of dietary supplementation by gym users. Method: This is a systematic review about the use of dietary supplements in gym goers conducted at the Medical Literature Analysis and Retrieval Sistem On-line (MEDLINE / PUBMED and Google academic, using the terms: food supplements, physical activity, body image The selection respected inclusion / exclusion criteria previously listed Results: 28 articles were selected, among them 12 met the requirements. The studies show that the prevalence of the use of supplements by gym users is higher in men than in women. reason observed for the use of dietary supplements was the search for muscle hypertrophy, with protein products being the most consumed among the supplements, however, it was observed that the prescription of such supplements is not performed by qualified professionals, being the gym instructors, followed by friends, store sellers, self-prescribing and lastly nutritionists. It was noted that the biggest consumers of dietary supplements are bodybuilders, since it is a modality that mixes muscle development as for better aesthetic results. Conclusion: Therefore, in order to reduce misuse, the presence of a specialized professional is necessary to work in gyms or in places where physical exercise is practiced, providing nutritional guidelines in order to improve the level of information and ensure safe use. of these products.
\end{abstract}

Keywords: Physical exercise; Food suplements; Nutrition; Bodybuilding. 


\begin{abstract}
Resumen
Introducción: Uno de los aspectos que ha definido a la sociedad de consumo es la creciente importancia que se le atribuye a la imagen corporal. Con ello, se consolidó la búsqueda de la práctica de actividades físicas en los gimnasios en sus diferentes modalidades en los distintos grupos de edad. Se sabe que la ingesta adecuada de nutrientes está relacionada con la mejora del rendimiento del organismo en relación al rendimiento deportivo. Con el fin de maximizar los resultados de los ejercicios, los practicantes deben buscar recursos para lograr el objetivo a corto plazo. Entre estos recursos, destacan los complementos alimenticios, de fácil adquisición, y muchas veces se utilizan de forma inadecuada con fines ergogénicos y puramente estéticos. Objetivo: evaluar, mediante una revisión, la prevalencia del uso de complementos dietéticos por parte de los usuarios de gimnasios. Método: Se trata de una revisión sistemática sobre el uso de suplementos dietéticos en asistentes al gimnasio realizada en el Medical Literature Analysis and Retrieval Sistem On-line (MEDLINE / PUBMED y Google académico, utilizando los términos: complementos alimenticios, actividad física, imagen corporal La selección Respetaron los criterios de inclusión / exclusión enumerados anteriormente Resultados: Se seleccionaron 28 artículos, de los cuales 12 cumplieron con los requisitos.Los estudios muestran que la prevalencia del uso de suplementos por parte de los usuarios de gimnasios es mayor en hombres que en mujeres.Razón observada para el uso de suplementos fue la búsqueda de la hipertrofia muscular, siendo los productos proteicos los más consumidos entre los suplementos, sin embargo, se observó que la prescripción de dichos suplementos no la realizan profesionales calificados, siendo los instructores de gimnasio, seguidos de amigos, vendedores de tiendas, -prescriptores y por último nutricionistas. Se notó que los mayores consumidores de suplementos dietéticos son los culturistas, ya que es una modalidad que mezcla el desarrollo muscular para obtener mejores resultados estéticos. Conclusión: Por tanto, para reducir el mal uso es necesaria la presencia de un profesional especializado que trabaje en gimnasios o en lugares donde se practique ejercicio físico, brindando pautas nutricionales con el fin de mejorar el nivel de información y garantizar un uso seguro de estos productos.
\end{abstract}

Palabras clave: Ejercicio físico; Suplementos alimenticios; Nutrición; Culturismo.

\title{
1. Introdução
}

Um dos aspectos que tem definido a sociedade de consumo é a crescente importância atribuída à imagem corporal. Com isso, consolidou-se nas diversas faixas etárias a busca pela prática de atividades físicas em academias nas suas diversas modalidades. Sabe-se que a ingestão adequada de nutrientes está relacionada com a melhora do rendimento do organismo frente ao desempenho esportivo. Em busca de potencializar resultados nos exercícios os praticantes são conduzidos à procura de recursos com o intuito de alcançar o objetivo em curto prazo Dentre esses recursos se destacam os suplementos alimentares, estes podem ser adquiridos facilmente, sendo muitas vezes utilizados de forma indevida com o propósito ergogênico e puramente estético (Almeida et al., 2019).

Sendo assim, associada a uma alimentação saudável, a utilização de recursos ergogênicos (RE), em sua maioria, carrega consigo benefícios adicionais, como prevenção, ou retardo de fadiga, melhoria do desempenho físico e estímulos hipertróficos, dentre outros. No entanto, a comercialização indiscriminada e prescrição não especializada desses produtos tornaram-se comum, repercutindo em consumo inadequado como também prejuízo e sobrecarga em alguns órgãos (Maximiano et al., 2017).

O papel do nutricionista é primordial para um adequado planejamento nutricional, pois juntamente com o indivíduo, norteia o balanço energético total, bem como distribuição e ajuste dos nutrientes, além de avaliar possíveis deficiências nutricionais, auxiliando na prevenção de lesões, fadiga muscular esquelética, ganho de massa muscular. Nesse interim, após o ajuste nutricional, a suplementação é uma ferramenta que pode ser usada para indivíduos que almejam desempenho físico, força e desenvolvimento de massa muscular, dos quais podem ser prescritas, aminoácidos, minerais, proteínas, carboidrato, dentre os compostos (Souza et al., 2020).

O interesse pela temática surgiu uma vez que a busca incessante pelo corpo perfeito vem crescente substancialmente atrelada a crescente utilização de suplementos alimentares, que muitas vezes é pautada sem a devida necessidade e também desprovida de orientação especializada, sendo assim o trabalho teve como objetivo investigar em qual público sua utilização se faz mais prevalente, qual o tipo mais consumido, além de verificar o conhecimento dos usuários acerca. 


\section{Metodologia}

Trata-se de uma revisão integrativa da literatura sobre o uso de suplementos dietéticos por praticantes de atividade física. A revisão integrativa emerge como uma metodologia que possibilita a síntese do conhecimento e a incorporação da aplicabilidade de resultados de estudos significativos na prática, além disso, aponta lacunas que precisam ser preenchidas com a realização de novos estudos com a finalidade de agregar resultados preliminares por meio da investigação crítica de temáticas relevantes no campo científico (Souza et al., 2017).

Nesse sentido, foi realizada nas bases de dados da Pubmed durante os meses de novembro de 2020 a janeiro de 2021. Foram empregados as palavras- chave: "suplementos alimentares", "nutrição" e "exercício físico" para realizar a busca dos artigos. No cruzamento das palavras, foi adotada a expressão booleana "AND” (inserção de duas ou mais palavras).

Os critérios de inclusão foram: 1) palavras chaves que possuíam relação com a temática em questão 2) estudos em português 3) disponibilidade de textos completos e gratuitos, 4) estudos com metodologia adequada ao objetivo proposto. O critério de exclusão se deu aos que não fossem originais, teses, dissertações, cartas ao editor, editoriais, monografias, estudos em animais e estudos com resultados conflitantes. Foram extraídos os dados a respeito de qual público sua utilização se faz mais frequente e principal motivação para o consumo.

\section{Resultados e Discussão}

A revisão foi baseada na consulta de artigos publicados no ano de 2012 a 2020. Conforme emprego das palavras- chave e base de dados apareceram 86 artigos que faziam referência à associação dos termos procurados. Após realizar a triagem baseada na avaliação de título e resumo restaram 27 estudos, em seguida, foram aplicados os critérios de inclusão e exclusão e restaram 12 artigos.

Os estudos mostram acerca da prevalência do uso suplementos por usuários de academias são maiores em homens do que em mulheres, estando em consonância com os achados de Weber et al., (2018), que ao realizar uma pesquisa com 185 pessoas de ambos os sexos verificou que apenas $20 \%$ dos homens e $13 \%$ das mulheres que faz uso de suplementos alimentares seguem indicação do profissional nutricionista. Notou-se que os maiores consumidores de suplementos alimentares são praticantes de musculação, visto que é uma modalidade que mescla o desenvolvimento muscular como para melhores resultados estéticos.

O principal motivo observado pela utilização de suplementos alimentares foi à busca pela hipertrofia muscular e estética, sendo os produtos proteicos os mais consumidos dentre os suplementos, visto que é um nutriente fundamental para o estímulo hipertrófico, no entanto, observou-se que a prescrição de tais suplementos não é realizada por profissionais habilitados e que a maioria dos frequentadores apresenta baixo conhecimento sobre suplementação, além disso, Rizzi et al., (2019) em seu estudo analisou que a maioria dos desportistas que faz uso de suplementos alimentares possuem hábitos inadequados, o que reforça a necessidade de um profissional capacitado para tal direcionamento, especialmente o nutricionista

Jost e Poll (2014) estudaram sobre o consumo de suplementos alimentares entre praticantes de atividade física em academias de Santa Cruz do Sul no estado de Rio Grande do Sul, e averiguaram que o público feminino (64,9\%) consumiu mais suplementos alimentares em comparação com sexo masculino (35,1\%), corroborando com os achados de Santos et al., (2013), o qual foi observado que grupo de mulheres alcança 51,8\% de utilização contra 41,2\% do grupo dos homens. Em contrapatida Silva et al., (2017) avaliou 70 esportistas e observou que o sexo masculino se sobressai quando o intuito é consumo de suplementos. 
Ademais, em um estudo realizado em duas academias de Palotinas- PR verificou que independente do tempo de prática de exercício, o sexo masculino tem marjorietariedade em se tratando de consumo de suplementos, comparando com as mulheres, que apresenta relação intrínseca com o tempo de prática (Weber et al., 2018).

Um estudo realizado por Bertulucci et al., (2012) foi visto que dentre as principais motivações que levaram o uso desses suplementos foi o aumento da massa muscular, disposição física e melhora da performance esportiva, sendo whey protein e creatina os mais consumidos, estando em acordo com Miragaia e Gomes (2020), que verificou em sua pesquisa que a principal razão que levou a utilização foi também hipertrofia muscular, com preponderância para o consumo de suplementos proteicos, já para as mulheres questões envolvidas na perda de peso e saúde, com destaque para o consumo de termogênicos, antioxidantes e complexos multivitamínicos, o que também foi observado em um estudo realizado por Silva et al., (2017) no qual comprovou que objetivos estéticos e hipertrofia como principal pretexto.

Cardoso e Leonhardt (2018) relatam que o uso abusivo e exagerado de proteínas além de não proporcionar benefícios adicionais pode gerar sobrecarga dos rins, ampliando a excreção de cálcio e diminuindo citratos na excreção urinária tornandoa mais ácida, podendo ocasionar alterações metabólicas, como aumento das taxas de colesterol total, triglicerídeos, e desregulação de secreções hormonais produzidos no intestino, o que também foi visto no estudo de Silva e Júnior (2020), que evidenciam que determinadas substâncias quando utilizadas de forma errônea causam danos hepáticos e renais

Segundo Gomes et al., (2018), os recursos podem ser utilizados para tratamento clínico ou isoladamente para melhorar o desempenho físico, no entanto, o acompanhamento por um profissional devidamente qualificado, se torna essencial para o correto direcionamento, afim de prevenir a ocorrência de danos ao organismo, bem como nortear a melhor conduta, por meio da avaliação de sua aplicabilidade individual.

Com o intuito de verificar os conhecimentos acerca dos suplementos alimentares, uma amostra constituída de 104

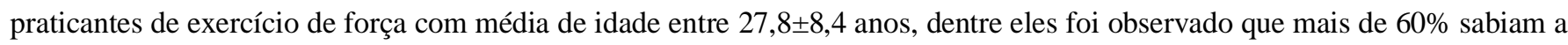
definição correta, e 55,7\% dos entrevistados afirmaram que o maior responsável pelas indicações foi o educador físico, no entanto, 87,5\% acreditam que isso é o papel do nutricionista, assemelhando-se com o estudo de Bertulucci (2012) os quais confirmam que $26 \%$ da indicação foi realizada por pessoas não habilitadas para tal função.

Em contrapartida, outra pesquisa realizada por Silva et al., (2018) com 41 praticantes de treinamento funcional avaliou os conhecimentos com relação ao consumo de suplementos alimentares e 53,7\% afirmaram não possuir, carecendo portanto de orientações adequadas. Sendo assim, Santos e Farias (2017) reforçam que o nutricionista é o profissional com maior grau de qualificação para prescrição de suplementos alimentares, sendo necessário portanto, a procura desse profissional para tal finalidade.

Reforçando resultados de achados anteriores, outra pesquisa realizada com 100 indivíduos praticantes de atividade física na cidade de Montes Claros- MG, buscou averiguar a prevalência de consumo de suplementos alimentares em praticantes de atividade, sendo 39,0\% mulheres e 61,0\% homens com idade entre 18 e 55 anos. Sendo assim, foi observado que $65 \%$ dos usuários faziam uso de tais recursos, destacando os suplementos proteicos como os mais consumidos, em razão do principal objetivo ser hipertrofia muscular. Além disso, notou-se que a maioria consumiu sem orientação especializada, o que confirma os achados de Moreira e Rodrigues (2014) que elucidam que a maioria dos frequentadores de academia faz uso de suplementação por indicação de instrutores de academias, profissionais de educação física e autoindicação.

\section{Considerações Finais}

Portanto, a fim de diminuir o uso indevido, se faz preciso a presença de profissional especializado para atuar em academias ou em locais em que haja prática de exercícios físicos, com o intuito de fornecer orientações nutricionais 
individualizadas a fim de melhorar o grau de informação, garantir segurança na utilização desses produtos.

\section{Referências}

Almeida, I. V., De Oliva Ribeiro, M. C., \& Freitas, R. F. (2019). Uso de suplementos alimentares e fatores associados em praticantes de atividade física de alta intensidade. RBNE - Revista Brasileira De Nutrição Esportiva, 12(76), 992-1004.

Cordeiro, S., Roza, I., Smolarek, A. D. C.,Mascarenhas, L. P. G. . (2020). Suplementos alimentares: Qual o conhecimento entre adolescentes? Revista Eletrônica Nacional De Educação Física, $10(15), 66$ - 74. https://doi.org/10.46551/rn2020101500040

Maximiano, C. M. B. F., \& Santos, L. C. (2017). Consumo de suplementos por praticantes de atividade física em academias de ginásticas da cidade de Sete Lagoas-MG. Revista Brasileira de Nutrição Esportiva, 11(61), 93-101.

Sousa L, M. M. S., Marques-Vieira, C. M. A., Severino, S. S., \& Antunes, A. V. 2017. Metodologia de revisão integrativa da literatura em enfermagem.

Santos, H. D., Oliveira, C. C. P. de, Freitas, A. K. C. de, \& Navarro, A. C. (2013). Consumo de suplementos alimentares por praticantes de exercício físico em academias de bairros nobres da cidade do Recife. RBNE - Revista Brasileira De Nutrição Esportiva, 7(40).

Silva, M. L., Teixeira, L. J. de M., Lima, J. S., Pereira, F. de O., \& Menezes, M. E. da S. (2017). Consumo de suplementos alimentares por praticantes de atividade física em academias de ginástica em Cuité-Paraíba. RBNE - Revista Brasileira De Nutrição Esportiva, 11(65), 644-653.

Bertulucci, K. N. B., Schembri, T., Pinheiro, A. M. M., \& Navarro, A. C. (2012). Consumo de suplementos alimentares por praticantes de atividade física em academias de ginástica em São Paulo. RBNE - Revista Brasileira De Nutrição Esportiva, 4(20).

Miragaia, D. A. M., \& Gomes, S. D. S. Perfil de Consumo de Suplementos Alimentares pelos Praticantes de Ginásio e Fatores que Influenciam a sua Utilização.

Cardoso, K. F., \& Leonhardt, V. (2018). Avaliação do consumo de suplementos proteicos por praticantes de musculação em uma academia de PlanaltinaDF. RBNE - Revista Brasileira De Nutrição Esportiva, 11(68), 1083-1091

Gomes, F. E. R.; Rodrigues, A. L. P.; Brito, L. C.; Soares, E. S. Análise do uso de suplementos alimentares e recursos ergogênicos por frequentadores de uma academia de Fortaleza-CE. Revista Brasileira de Nutrição Esportiva, 12(69), 100-108, 2018.

dos Santos, E. A., \& Pereira, F. B. (2017). Conhecimento sobre suplementos alimentares entre praticantes de exercício físico. RBNE - Revista Brasileira De Nutrição Esportiva, 11(62), 134-140.

Santos, A. V., \& Farias, F. O. (2017). Consumo de suplementos nutricionais por praticantes de atividades físicas em duas academias de Salvador-BA. RBNE Revista Brasileira De Nutrição Esportiva, 11(64), 454-461.

da Silva, C. F., Vieira da Fonseca, T. C., de Menezes Filho, H. J., Veloso, L. F., Maia, M. B., \& Freitas, R. F. (2018). Conhecimento acerca da alimentação saudável e consumo de suplementos alimentares por praticantes do treinamento funcional. RBNE - Revista Brasileira De Nutrição Esportiva, 12(74), 757-765

Silva, R. P. D. Q. C., dos Santos Vargas, V., \& Lopes, W. C. (2017). Consumo de suplementos alimentares por praticantes de atividade física em academias. Revista Brasileira de Nutrição Esportiva, 11(65), 584-592.

Moreira, F. P., \& Rodrigues, K. L. (2014). Conhecimento nutricional e suplementação alimentar por praticantes de exercícios físicos. Revista Brasileira de Medicina do Esporte, 20(5), 370-373.

Rizzi, T., Hoerlle, E., Braga, K. D., Pretto, A. D. B., \& Pastore, C. A. (2019). Consumo alimentar e imagem corporal em desportistas do município de PelotasRS. RBNE - Revista Brasileira De Nutrição Esportiva, 13(77), 35-44.

Silva, F. Q., Mota, K. P. S., da Cunha, M. D. S. B., \& da Silva, D. C. G. (2019). Consumo de suplementos nutricionais em praticantes de atividade física de uma academia do oeste da Bahia. Revista científica da Faminas, 14(1).

Weber, M. G., Brandt, R., Olivoto, R. R., \& Flores, L. F. (2018). Musculação e suplementação: perfil dos consumidores de suplementos alimentares nas academias de Palotina-PR. RBNE - Revista Brasileira De Nutrição Esportiva, 12(75), 852-861. 